\title{
ANTIBIOTIC RESISTANCE PATTERN OF BACTERIAL ISOLATES FROM POST- TRACHEOSTOMISED PATIENTS ATTENDING TERTIARY CARE HOSPITAL IN NEPAL
}

Eliza Thapa, Roshan Thapa, Anjana Singh, Bharat Mani Pokharel, Upendra Devkota

Journal of Institute of Science and Technology Volume 21, Issue 1, August 2016 ISSN: 2469-9062 (print), 2467-9240(e)

Editors:

Prof. Dr. Kumar Sapkota

Prof. Dr. Armila Rajbhandari

Assoc. Prof. Dr. Gopi Chandra Kaphle

JIST, 21 (1), 129-132 (2016)

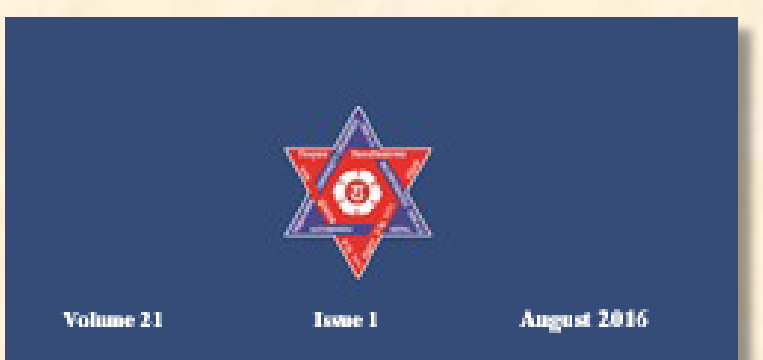

JOURNAL OF INSTITUTE OF SCIENCE AND TECHNOLOGY

Institute of Selence and Teehnology

Tribhuvan University

Kirtipur, Kathmanda, Nepal

Published by:

Institute of Science and Technology

Tribhuvan University

Kirtipur, Kathmandu, Nepal 


\title{
ANTIBIOTIC RESISTANCE PATTERN OF BACTERIAL ISOLATES FROM POST- TRACHEOSTOMISED PATIENTS ATTENDING TERTIARY CARE HOSPITAL IN NEPAL
}

\author{
Eliza Thapa ${ }^{1, *}$, Roshan Thapa ${ }^{1}$, Anjana Singh ${ }^{1}$, Bharat Mani Pokharel ${ }^{2}$, Upendra Devkota ${ }^{3}$ \\ ${ }^{1}$ Central Department of Microbiology, Tribhuvan University, Kirtipur, Nepal \\ Institute of Medicine, Tribhuvan University, Maharajgunj, Kathmandu, Nepal \\ National Institute of Neurological and Allied Sciences, Bansbari, Kathmandu, Nepal \\ ${ }^{*}$ Corresponding email: thapaeliza2804@gmail.com
}

\begin{abstract}
The bacterial profile and their drug susceptibility pattern was studied in post-tracheostomised patients admitted in National Institute of Neurological and Allied Sciences. Identification of organisms was done by standard microbiological techniques and antibiogram was performed by Kirby Bauer disc diffusion method according to Clinical and Laboratory standard (CLSI) guidelines. Significant growth was observed in 58.6\% samples with polymicrobial growth in $19.5 \%$ samples among the total positive cultures. Pseudomonas aeruginosa was the predominant organism (34.2\%) followed by Acinetobacter spp. (31.8\%), Escherichia coli (8.9\%), Klebsiella pneumoniae (6.0\%), Citrobacter fruendii (5.6\%), Klebsiella oxytoca (4.0\%), Providencia spp. and Staphylococcus aureus (2.8\% each), Proteus mirabilis (2.4\%) and Proteus vulgaris $(1.2 \%)$. More than $90 \%$ of Gram negative bacterial isolates were found resistant to Ampicillin, Cefixime, Cephotaxime. The most effective drugs against $S$. aureus were Vancomycin, Rifampicin, Amoxycillin and Clavulanic acid showing 100\% sensitivity. Highest resistance rate was observed for Ampicillin (85.71\%) and low for Gentamicin (14.28\%). Findings of this study show emerging threat of multidrug resistant bacteria. Thus, periodic monitoring and assessing drug susceptibility pattern of bacteria and rational use of antibiotics in post tracheostomised patients were recommended.
\end{abstract}

Key words: Kirby Bauer disc diffusion, Acinetobacter, Pseudomonas aeruginosa

\section{INTRODUCTION}

Tracheostomy is an invasive procedure aimed to provide a secure airway to ventilate and aspirate the patient in the critical care setting (Pignatti et al. 2009). It is performed in many clinical cases such as decreased level of consciousness, poor airway protective reflexes, severe alterations in physiology associated with trauma and medical illness and the most common being prolonged respiratory failure (Durbin 2010). Tracheostomy is associated with a number of clinical complications out of which airway colonisation and infection is the most frequent complication in patients with chronic tracheaostomy (Apostolopoulou et al. 2003). The colonisation route is either endogenous or exogenous which generally causes lower airway infection such as tracheobronchitis and bronchopneumonia. Nosocomial bacteria such as Pseudomonas aeruginosa, Acinetobacter spp., methicillin resistant Staphylococcus aureus,
Serratia spp. and members of Enterobacteriaceae are the frequently encountered infection causing bacteria. However, others like Streptococcus pneumoniae, Moraxella catarrhalis, Haemophilus influenzae, and methicillin sensitive $S$. aureus also colonise and infect the lower respiratory tract (Ionas et al. 2001, Morar et al. 2002, Erdem et al. 2008). This work was undertaken to assess the antibiotic susceptibility of bacteria isolated from samples associated with tracheostomised patients.

\section{MATERIALS AND METHODS}

This study was conducted at National Institute of Neurological and Allied Sciences, Bansbari, Kathmandu, Nepal from March 2014 to August 2014. A total of 350 clinical specimens including tracheal aspirates (180), urine (73), blood (22), pus (13), wound swabs (4), Cerebrospinal fluid (23), Catheter tip (13), CVP tip (19), Foley's tip (1) and ICP tips (2) from tracheostomised 
patients that were sent to microbiology laboratory for routine analysis were processed via standard microbiological procedure. Identification of organisms was done on the basis of colony characteristics, staining, biochemical tests such as catalase, oxidase, sulfide indole motility, citrate, urea hydrolysis, triple sugar iron agar test and coagulase tests. Antibiotic susceptibility test was performed using the Kirby Bauer disk diffusion method and results were interpreted according to CLSI guidelines (CLSI 2007). The approval to conduct this work was obtained from the hospital.

\section{RESULTS}

Among the samples processed, significant bacterial growth was observed in $205(58.6 \%)$ samples with polymicrobial growth in $19.5 \%$ of the total positive cultures. From these 205 positive samples 248 bacterial isolates were obtained out of which $2.8 \%$ were Gram positive cocci and remaining $97.2 \%$ were Gram negative rods and coccobacilli. Gram positive cocci were identified as $S$. aureus whereas majority (34.2\%) of the Gram negative isolates were identified as $P$. aeruginosa (Table 1).
Table 1. Percentage of bacterial isolates among positive cases

\begin{tabular}{cc}
\hline Bacterial organisms & Frequency (\%) \\
\hline P. aeruginosa & 34.2 \\
Acinetobacter spp. & 31.8 \\
E. coli & 8.9 \\
K. pneumonia & 6.0 \\
C. fruendii & 5.6 \\
K. oxytoca & 4.0 \\
Providencia spp. & 2.8 \\
S. aureus & 2.8 \\
P. mirabilis & 2.4 \\
P. vulgaris & 1.2 \\
\hline Total & $\mathbf{1 0 0}$ \\
\hline
\end{tabular}

Antibiotic resistance profiles revealed that majority of bacterial isolates were resistant to multiple antibiotics. The Gram negative bacteria were observed to be highly resistant to Ampicillin, Cefexime, Cephotaxime and Cotrimoxazole. Acinetobacter spp. showed high resistance to multiple antibiotics like Ampicillin, Cephotaxime, Ciprofloxacin, Cotrimoxazole and PiperacillinTazobactam but high susceptibility (more than 97\%) was shown towards Polymyxin B and Colistin (Table 2).

Table 2: Antibiotic susceptibility profile of Gram negative bacteria

\begin{tabular}{lcccccccccc}
\hline Antibiotics & Conc. & \multicolumn{8}{c}{ Percent of isolates showing antibiotic resistance } \\
& $(\mathbf{m c g})$ & PS & KO & KP & EC & CF & P & PV & PM & AC \\
\hline Ampicillin & 10 & 100 & 90 & 100 & 90.9 & 100 & 100 & 100 & 66.67 & 100 \\
Amikacin & 30 & 8.23 & 50 & 46.67 & 18.18 & 57.18 & 100 & 66.67 & 0 & 86.1 \\
Carbenicillin & 100 & 43.53 & - & - & - & - & - & - & - & - \\
Cefipime & 30 & 78.86 & - & - & - & - & - & - & - & - \\
Cefixime & 5 & - & 100 & 100 & 69.23 & 92.86 & 100 & 100 & 0 & - \\
Cephotaxime & 30 & 90.59 & 80 & 100 & 68.18 & 92.85 & 100 & 100 & 0 & 97.5 \\
Ciprofloxacin & 5 & 77.64 & 80 & 60 & 69.23 & 57.14 & 42.85 & 100 & 0 & 92.4 \\
Colistin & 10 & - & - & - & - & - & - & - & - & 1.3 \\
Cotrimoxazole & 25 & 88.24 & 90 & 86.67 & 72.73 & 78.57 & 100 & 100 & 0 & 98.7 \\
Gentamicin & 10 & 11.76 & 50 & 53.33 & 31.81 & 71.42 & 85.71 & 0 & 0 & 83.5 \\
Imipenem & 10 & 3.52 & 0 & 6.67 & 9.09 & 0 & 0 & 0 & 0 & 39.2 \\
Nalidixic acid & 30 & - & - & - & 88.88 & - & - & - & - & - \\
Nitrofurantoin & 300 & - & - & - & 0 & - & - & - & - & - \\
Piperacillin- & $100 / 10$ & 60 & 30 & 40 & 40.9 & 28.57 & 85.71 & 33.33 & 0 & 92.4 \\
Tazobactam & & & & & & & & & & \\
Polymyxin B & 300 & - & - & - & - & - & - & - & - & 0 \\
\hline
\end{tabular}

PS: P. aeruginosa; KO: K. oxytoca; KP: K. pneumoniae; EC: E. coli; SA: S. aureus; CF: C. fruendii; P: Providencia spp; PV: P. vulgaris; PM: P. mirabilis; AC: Acinetobacter spp.; (-): antibiotic not used for particular organism 
Most of the S. aureus isolates were found to be resistant to Ampicillin while moderate numbers were resistant to Cotrimoxazole, Cephoxitin and Erythromycin. Few isolates were found resistant against Gentamicin whereas none were resistant against Vancomycin, Rifampicin and Amoxycillin-Clavulanic acid (Table 3).

Table 3: Antibiotic susceptibility profile of $S$. aureus

\begin{tabular}{lcc}
\hline \multicolumn{1}{c}{ Antibiotics } & Conc $(\mathbf{m c g})$ & Percent of isolates showing resistance \\
\hline Ampicillin & 10 & 85.71 \\
Amoxycillin clavulanic acid & 30 & 0 \\
Cephoxitin & 30 & 57.14 \\
Ciprofloxacin & 5 & 42.85 \\
Cotrimoxzole & 25 & 57.14 \\
Erythromycin & 15 & 63.42 \\
Gentamicin & 10 & 14.28 \\
Rifampicin & 5 & 0 \\
Vancomycin & 30 & 0 \\
\hline
\end{tabular}

\section{DISCUSSION}

Results of this study showed that the bacterial growth is high in number, which is in the harmony with another study conducted in the same hospital that reported $60.88 \%$ positive growth (Bhandari et al. 2015). Polymicrobial growth was reported in $19.5 \%$ samples. Most of the polymicrobial growths in this study were associated with Acinetobacter spp. and $P$. aeruginosa, the two frequently acquired nosocomial pathogens. This high growth rate with polymicrobial growth may be either due to the fact that the patients were critically ill and thus had poor immune system to combat against pathogenic microbes or due to presence of these bacteria in the hospital environment.

The dominant isolates accounted were Gram negative bacteria. High prevalence of Gram negative rods in tracheostomised patients was also reported in other study (Siddiqui et al. 2011). P. aeruginosa was the predominant organism which is also analogous to other studies (Morar et al. 2000, Nseir et al. 2002). Factors such as their ubiquity in hospital environment, ability to form incredibly hard biofilms on many seemingly uninhabitable surfaces and calcium alginate capsules may have contributed to the highest prevalence of $P$. aeruginosa (Inglis et al. 1989).

Besides, some of the isolated bacteria were resistant to more than one antibiotic. It was found that more than $90 \%$ of Gram negative bacterial isolates were resistant to Ampicillin, Cefixime, Cephotaxime. Low resistance was observed for Imipenem with resistance rate ranging between 3.52-9.09\%. For aminoglycosides such as Amikacin and Gentamicin, the resistance rate was between 8.23-100\%. Resistance for Ciprofloxacin was observed between $42.85-100 \%$, for Cotrimoxazole between 57.14-100\% and for Piperacillin-Tazobactam between $28.57-85.71 \%$. Similar pattern of degree of resistance was witnessed in different studies (Kumari et al. 2007, Mohammadimehr \& Feizabadi 2011). Contrary, a study by Hossein et al. (2012) reported resistance of $45-75 \%$ to Cefixime. Gram negative organisms adopt different resistance mechanism to neutralize the affects of many commonly used antibiotics. This may be contributed by the production of $\beta$-lactamases primarily ESBL, AmpC enzymes, metallo-carbapenemases, aminoglycoside modifying enzymes, loss of porin proteins and the presence of efflux pumps like MexABOpr M. An increase in antibiotic resistance among Gram negative bacterial isolates has been a great concern in hospitals. Overuse of antimicrobial agents and problems with infection control practices have led to the development of multidrug resistant Gram negative bacterial infections (Falagas et al. 2006).

A notorious nosocomial bug, Acinetobacter spp. showed high resistance to a number of antibiotics. This resistance mechanism is furnished via altered penicillin-binding proteins, low/decrease permeability of the outer membrane to antibiotics or increase in the active efflux of the antibiotics, target site mutations and inactivation (Jain \& Danziger 2004). The type of species, antibiotic and geographical location is also conducive to resistance mechanism (Vila et al. 2002, Jain \& Danziger 2004).

\section{CONCLUSION}

We conclude that there is a significant prevalence of Gram negative microorganisms in post tracheostomised patients with $P$. aeruginosa being the most common. Most of the bacterial isolates were resistant to multiple antibiotics, which is a threat to the emergence of Multi Drug Resistant bacteria making treatment of infections extremely difficult. Hence, early detection of infection, empirical use of antibiotics and restriction in use of 
broad spectrum antibiotics are obligatory. Since majority of isolates are nosocomial pathogens, aggressive cleaning and monitoring of hospital environment is necessary to minimize the spread of theses bugs to other immune-compromised patients.

\section{ACKNOWLEDGEMENTS}

The authors would like to thank all the staff of Pathology Department of National Institute of Neurological and Allied Sciences and the patients for their generous help and support.

\section{REFERENCES}

Apostolopoulou, E., Bakakos, P., Katostaras, T. and Gregorakos L. 2003. Incidence and risk factors for ventilator associated pneumonia in 4 multidisciplinary Intensive Care Units in Athens, Greece. Respiratory Care 48(7): 681-688.

Bhandari, P., Thapa, G., Pokhrel, B. M. and Devkota, U. 2015. Nosocomial Isolates and Their Drug Resistant Pattern in ICU Patients at National Institute of Neurological and Allied Sciences, Nepal. International Journal of Microbiology Article ID 572163, 6, doi:10.1155/2015/572163

Durbin, C. G. 2010. Tracheostomy: why, when, and how? Respiratory Care 55(8): 1056-1068.

Erdem, I., Ozgultekin, A., Inan, A. S., Dincer, E., Turan, G., Ceran, N., et al. 2008. Incidence, etiology and antibiotic resistance patterns of Gram negative microorganisms isolated from patients with VAP in a medical surgical unit of a Teaching Hospital in Istanbul, Turkey. Journal of Infectious Disease 61: 339-342.

Falagas, M. E., Koletsi, P. K. and Bliziotis, I. A. 2006. The diversity of definitions of multidrug resistant (MDR) and pandrug resistant (PDR) Acinetobacter baumannii and Pseudomonas aeruginosa. Journal of Medical Microbiology 55: 1619-1629.

Clinical and Laboratory Standards Institute (CLSI) 2007. Performance standards for antimicrobial susceptibility testing, 17th Informational Supplement CLSI M100S17, Clinical and Laboratory Standards institute, Wayne, Pa, USA.

Hossein, K., Dashti, Khavidaki, S., Shahidi, M., Abdollahi, A., Jafari, S., Jahangard Rafsanjani, Z. et al. 2012. Changes in Gram negative microorganisms' resistance pattern during 4 years period in a referral teaching hospital; a surveillance study. DARU Journal of Pharmaceutical Sciences 20: 28.
Inglis, T. J., Millar, M. R., Jones, G. and Robinson, D. A. 1989. Tracheal tube biofilm as a source of bacterial colonization of the lung. Journal of Clinical Microbiology 27: 2014-2018.

Ionas, M., Ferrer, R., Angrill, J., Ferrer, M. and Torres, A. 2001. Microbial investigation in ventilator associated pneumonia. European Respiratory Journal 17: 791-801.

Jain, R. and Danziger, L. H. 2004. Multidrug resistant Acinetobacter infections: An emerging challenge to clinicians. Annals of Pharmacotherapy 38: 1449-1459.

Kumari, H. B. V., Nagarathna, S. and Chandramuki, A. 2007. Antimicrobial resistance pattern among aerobic Gram negative bacilli of lower respiratory tract specimens of intensive care unit patients in a Neurocentre. Indian Journal of Chest Diseases and Allied Sciences 49: 1922.

Mohammadimehr, M. and Feizabadi, M.M. 2011. Antimicrobial resistance pattern of Gram negative bacilli isolated from patients at ICUs of Army hospitals in Iran. Iranian Journal of Microbiology 3(1): 26-30.

Morar, P., Singh, V., Makura, Z., Jones, A. S., Baines, P. B., Selby, A., et al. 2002. Oropharyngeal carriage and lower airway colonisation/infection in 45 tracheotomised children. Thorax 57: 1015-1020.

Nseir, S., Di Pompeo, C., Pronnier, P., Beague, S., Onimus, T., Saulnier, F., et al. 2002. Nosocomial tracheobronchitis in mechanically ventilated patients: incidence, aetiology and outcome. European Respiratory Journal 20: 1483-1489.

Pignatti, P., Balestrino, A., Herr, C., Bals, R., Moretto, D., Corradi, M., et al. 2009. Tracheostomy and related hostpathogen interaction are associated with airway inflammation as characterized by tracheal aspirate analysis. Respiratory Medicine 103: 201-208.

Siddiqui, Z. R., Ahmed, E., UdDin, N. and Uz Zaman, K. 2011. Antimicrobial sensitivity of lower respiratory tract infections in tracheostomised severe head injury patients. Annals of Pakistan Institute of Medical Sciences 7(2): 52-56.

Vila, J., Ribera, A., Marco, F., Ruiz, J., Mensa, J., Chaves, J., et al. 2002). Activity of clinafloxacin, compared with six other quinolones, against Acinetobacter baumannii clinical isolates. Journal of Antimicrobial Chemotherapy 49: 471-477. 